\title{
The Neural Correlates of Persuasion: A Common Network across Cultures and Media
}

\author{
Emily B. Falk ${ }^{1}$, Lian Rameson ${ }^{1}$, Elliot T. Berkman ${ }^{1}$, Betty Liao ${ }^{1}$, \\ Yoona Kang ${ }^{2}$, Tristen K. Inagaki ${ }^{1}$, and Matthew D. Lieberman ${ }^{1}$
}

\begin{abstract}
Persuasion is at the root of countless social exchanges in which one person or group is motivated to have another share its beliefs, desires, or behavioral intentions. Here, we report the first three functional magnetic resonance imaging studies to investigate the neurocognitive networks associated with feeling persuaded by an argument. In the first two studies, American and Korean participants, respectively, were exposed to a number of text-based persuasive messages. In both Study 1 and Study 2, feeling persuaded was associated with increased activity in posterior superior temporal sulcus bilaterally, temporal pole bilaterally, and dorsomedial prefrontal cortex. The findings suggest a discrete set of underlying mechanisms in the moment that the persuasion
\end{abstract}

process occurs, and are strengthened by the fact that the results replicated across two diverse linguistic and cultural groups. Additionally, a third study using region-of-interest analyses demonstrated that neural activity in this network was also associated with persuasion when a sample of American participants viewed video-based messages. In sum, across three studies, including two different cultural groups and two types of media, persuasion was associated with a consistent network of regions in the brain. Activity in this network has been associated with social cognition and mentalizing and is consistent with models of persuasion that emphasize the importance of social cognitive processing in determining the efficacy of persuasive communication.

\section{INTRODUCTION}

Persuasion is a common social exchange in which one person or group attempts to convince another of its beliefs, desires, or behavioral intentions. Aristotle devoted an entire volume to the mechanisms of persuasion, attesting to the enduring significance of this type of human interaction (Aristotle, 1926). He suggested that an individual might be persuaded as a result of the logic of an argument $(\log O s)$, the emotional appeal of an argument (pathos), or factors related to the source of the persuasive message (ethos). Reasoning, emotion, and characteristics of the message source have continued to be central factors examined in modern models of persuasion and attitude change, although the terminology used to describe these factors has changed to include ideas such as cognitive elaboration, affective appeal, and perceived similarity to the message source (Crano \& Prislin, 2008; Albarracin, Johnson, \& Zanna, 2005; Johnson, Maio, \& Smith-McLallen, 2005; Eagly \& Chaiken, 1993; Stayman \& Batra, 1991; Chaiken, Liberman, \& Eagly, 1989; Petty \& Cacioppo, 1986; Zajonc \& Markus, 1982).

Because behavioral methods can only assess one measure at a time, it has not been possible to assess the simultaneous cognitive, affective, and social processes that may

${ }^{1}$ University of California, Los Angeles, ${ }^{2}$ Yale University, New Haven, CT occur in concert during persuasion attempts or determine the relative priority with which each contributes to effective persuasion. Limitations of introspective self-reports are well documented (Wilson \& Schooler, 1991; Nisbett \& Wilson, 1977); even implicit measures, which circumvent self-report difficulties, are incapable of assessing persuasion processes at the moment they are occurring without simultaneously imposing a concurrent cognitive task. Using behavioral methods, attempts to measure persuasion while it is actually occurring would almost certainly alter the persuasion process itself.

Although having limitations of its own, fMRI has some important advantages in the study of persuasion and, therefore, is an important complement to existing methodologies. Critically, fMRI allows the neurocognitive processes associated with persuasion to be assessed as they unfold, and thus, the processes operative at the moment of persuasion can be identified without interruption. Additionally, fMRI is not constrained to examine a single process at a time. Because there are well-established neural networks associated with cognitive, affective, and social processes (Lieberman, 2007; Cabeza \& Nyberg, 2000), the presence or absence of each of these processes can be examined simultaneously. Based on previous persuasion research, a number of candidate neurocognitive networks that might contribute to the persuasion process were identified. If argument logic, emotional appeal, and message source characteristics are factors that impact persuasion under 
different circumstances, as both Aristotle and modern research suggest, then deliberative reasoning (associated with activity in the lateral prefrontal and parietal cortices), emotional processing (associated with activity in the limbic system), and social cognition (associated with activity in dorsomedial prefrontal cortex [DMPFC], posterior superior temporal sulcus [pSTS], and temporal poles [TP]), respectively, are psychological processes that should relate to experiencing an argument as persuasive (Crano \& Prislin, 2008; Lieberman, 2007; Albarracin et al., 2005; Johnson et al., 2005; Campbell \& Babrow, 2004; Cabeza \& Nyberg, 2000; Eagly \& Chaiken, 1993; Stayman \& Batra, 1991; Chaiken et al., 1989; Petty \& Cacioppo, 1986; Zajonc \& Markus, 1982). In addition, memory encoding (Stayman \& Batra, 1991; Chaiken et al., 1989) and self-referential processing (Meyers-Levy \& Peracchio, 1995), the former of which has been associated with activity in the medial temporal lobe and left ventrolateral prefrontal cortex (VLPFC), and the latter of which has been associated with activity in medial prefrontal cortex and precuneus/posterior cingulate, may contribute to persuasion effects under some circumstances.

In this article, we report three functional magnetic resonance imaging (fMRI) studies that begin to elucidate the neurocognitive networks associated with feeling persuaded across two different cultural/linguistic groups (Americans and Koreans), and across two different categories of media conveying persuasive messages (text-based arguments and video-based commercials). We used a within-subjects design allowing us to correlate the individual experience of persuasion with neural activity in order to explore which of the above networks and regions are reliably associated with persuasion across individuals. We also conducted between-groups analyses to examine these effects across two cultural groups in order to identify points of convergence and divergence as a function of culture.

\section{METHODS (STUDIES 1 AND 2)}

In a first study, 15 American participants simultaneously read and heard arguments related to a number of different objects and activities (e.g., flossing, blood donation) during an fMRI scanning session. Participants were reminded of each argument and were asked to rate its persuasiveness shortly after exiting the scanner. In order to identify the neural mechanisms associated with finding an argument persuasive, we compared blood oxygenation level dependent (BOLD) response as participants were exposed to trials that they subsequently rated as persuasive relative to BOLD response during trials that they subsequently rated as unpersuasive.

Numerous social science phenomena studied exclusively within Western countries (i.e., North America, Western Europe) were once thought to be universal until examination of those phenomena in East Asian populations revealed strong cross-cultural differences (Nisbett, 2003; Markus \& Kitayama, 1991). Likewise, persuasive ef- fects have been shown to differ along cultural dimensions such as individualism/collectivism (Uskul, Sherman, \& Fitzgibbon, 2009; Khaled, Ronald, Noble, \& Biddle, 2008; Kreuter \& Mcclure, 2004; Aaker \& Williams, 1998). We therefore conducted a second study within a cultural neuroscience framework (Chiao \& Ambady, 2007) using the same methodology but with a culturally different sample to replicate the findings and examine whether they would generalize across cultural boundaries. Topics and wording were also reviewed by individuals from America and Korea to confirm similar relevance of the topics and presentation in each culture.

\section{Participants (Study 1)}

Fifteen participants ( 7 women; mean age $=20.75$ years, $S D=3.21$ ) were recruited from the UCLA subject pool and through mass emails and posted fliers, and received either course credit or financial compensation for their participation. All participants were right-handed, European American, born and raised in the United States, and spoke English as their first language. Participants also met the following criteria related to fMRI safety: (1) were not claustrophobic; (2) had no metal in their bodies (other than tooth fillings); and (3) were not pregnant or breastfeeding. Potential participants were excluded if they were currently taking any psychoactive medication.

\section{Participants (Study 2)}

Fourteen participants ( 11 women; mean age $=22.06$ years, $S D=3.96$ ) were recruited from the UCLA subject pool and from mass emails and posted fliers, and received either course credit or financial compensation for their participation. All participants were right-handed, Asian, were born and raised for more than half of their lifetime in Korea, and spoke Korean as their first language. Participants met identical safety criteria to Study 1 .

\section{Materials (Studies 1 and 2)}

Materials for Studies 1 and 2 included text-based persuasive arguments about 20 different objects and activities. Each set of arguments about a given object or activity consisted of five phrases (one main argument and four supporting phrases), resulting in 100 total phrases across the 20 blocks. Phrases were developed by a team of American and Korean researchers to minimize cultural biases. The phrases were selected to be highly comprehensible, range in level of persuasiveness, and pertain to objects and activities about which people were likely to have weak initial attitudes. In Study 1, all phrases and instructions were presented in English. In Study 2, phrases and instructions were presented in Korean. Individual difference measures relevant to culture, including individualism/collectivism (Singelis, Triandis, Bhawuk, \& Gelfand, 1995; Triandis, 1995) and 
independence/interdependence (Singelis, 1994), were collected from each participant.

\section{Translation (Study 2)}

Instructions and stimuli were all translated by a native Korean-speaking professional translator with prior experience working in and translating for the psychological sciences. After discussion of the aims of the research, the primary translator provided a first draft translation, which was reviewed by a bilingual member of the research team, and corrections were made in line with the scientific goals of the study. After approval of all changes by the primary translator, a second, native English-speaking translator was hired to provide a back-translation to correct any errors. All mismatches were addressed and the final translation was approved by the primary translator, the secondary translator, and a bilingual reviewer on the research team.

\section{Procedure (Studies 1 and 2)}

While in an fMRI scanner, each participant viewed all 20 blocks (100 phrases) arranged into four runs, with order of the runs counterbalanced across subjects. Each run contained five randomly ordered blocks, with each block pertaining to a different object or activity. Each block began with one argument phrase followed by four supporting phrases, for a total of five phrases about any given object or activity. Blocks ranged from 33 to $61 \mathrm{sec}$ in English, and 33 to $57 \mathrm{sec}$ in Korean, and were separated by a 15 -sec fixation-cross baseline period. Participants were instructed to read each phrase, to consider each phrase carefully, and were told that they would later be asked some questions about what they had read (persuasion was not mentioned at any point prior to the postscan questionnaires). The instructions were repeated before each run. In order to control for reading speed, each phrase displayed on the screen was also presented aurally via prerecorded cues. Following the scanner session, participants were asked to rate whether each group of phrases as a whole was persuasive on a 4-point scale ("This paragraph, as a whole, is PERSUASIVE: $1=$ Disagree Strongly $2=$ Disagree Somewhat $3=$ Agree Somewhat $4=$ Agree Strongly"). Participants also rated the extent to which they believed that the arguments were based on information and based on feelings, using the same 4-point scale. Aside from language, Korean and American participants completed an identical task.

\section{Data Acquisition and Analysis}

Imaging data were acquired using a Siemens Allegra 3-Tesla head-only MRI scanner at the UCLA Ahmanson-Lovelace Brainmapping Center. Head motion was minimized using foam padding and surgical tape; goggles were also fixed in place using surgical tape connecting to the head coil and scanner bed. A set of high-resolution structural T2weighted echo-planar images were acquired coplanar with the functional scans (spin-echo; TR $=5000 \mathrm{msec}$; TE $=$ 33 msec; matrix size $=128 \times 128 ; 36$ axial slices; FOV $=$ $20 \mathrm{~cm}$; voxel size $=1.6 \times 1.6 \times 3.0 \mathrm{~mm}$ ). Four functional runs were recorded (echo-planar T2-weighted gradientecho, $\mathrm{TR}=2000 \mathrm{msec}, \mathrm{TE}=25 \mathrm{msec}$, flip angle $=90^{\circ}$, matrix size $=64 \times 64,36$ axial slices, $\mathrm{FOV}=20 \mathrm{~cm}$; voxel size $=3.1 \times 3.1 \times 3.0 \mathrm{~mm}$ ) lasting 328, 312, 310, and $298 \mathrm{sec}$, respectively, for Study 1, and 321, 302, 307, and $295 \mathrm{sec}$, respectively, for Study 2.

The data were analyzed using Statistical Parametric Mapping (SPM5, Wellcome Department of Cognitive Neurology, Institute of Neurology, London, UK). Images were realigned to correct for motion, slice timed, normalized into standard stereotactic space (Montreal Neurological Institute $[\mathrm{MNI}]$ ), and smoothed with an 8-mm Gaussian kernel, full width at half maximum. The task was modeled for each participant using a weighted linear contrast, comparing neural responses during arguments rated persuasive (rating of 3 or 4 ) vs. unpersuasive (rating of 1 or 2); the subjects' primary ratings were used to sort the blocks (persuasive or not) for each individual and then a $1,-1$ dummy variable was used for persuasive or not. All analyses were run at a threshold of $p<.001$, uncorrected, with a 5 -voxel extent threshold. All coordinates are reported in MNI space.

\section{RESULTS (STUDIES 1 AND 2)}

\section{Study 1: Persuasiveness of Text-based Messages (American Participants)}

In examining the neural response to persuasive compared to unpersuasive arguments in American participants viewing text-based messages, DMPFC, bilateral pSTS, and bilateral TP, were each more active during the presentation of arguments that were subsequently rated as persuasive compared to arguments that were rated as unpersuasive (Table 1A; Figure 1). These three regions have been repeatedly observed to be coactive in "theory-of-mind" and mentalizing studies (Frith \& Frith, 2003) and do not typically appear together during other kinds of processing (Cabeza \& Nyberg, 2000). Mentalizing refers to the ability to infer the mental states (desires, intentions and beliefs) of other people, and has been extensively studied in the brain (Frith \& Frith, 2003).

Bilateral medial temporal lobe and left VLPFC, regions often implicated in memory processes (Badre \& Wagner, 2007; Wagner et al., 1998), were also more active to persuasive, relative to unpersuasive, arguments. Visual cortex was the only other brain region where activity was greater during persuasive than unpersuasive passages.

\section{Study 2: Persuasiveness of Text-based Messages (Korean Participants)}

The results of Study 2 were remarkably consistent with Study 1 (Figure 1; Table 1A). In fact, there was no brain 
Table 1. Brain Regions Showing Differences in Brain Activity for Persuasive Relative to Unpersuasive Passages (Thresholded at $p<$ .001, Uncorrected, 5 Voxel Extent)

A. Brain Regions Showing Increased Activity for Persuasive Relative to Unpersuasive Passages

Study 1

\begin{tabular}{|c|c|c|c|c|c|c|c|}
\hline Phrases (American) & Brodmann's Area & Laterality & $x$ & $y$ & $z$ & $t$ & Vox \\
\hline DMPFC & 9 & $\mathrm{~L}$ & -14 & 66 & 28 & 4.69 & 15 \\
\hline pSTS & 22 & $\mathrm{~L}$ & -58 & -36 & 4 & 4.82 & 418 \\
\hline pSTS & 22 & $\mathrm{R}$ & 60 & -26 & -2 & 5.19 & 356 \\
\hline $\mathrm{TP}$ & $21 / 38$ & $\mathrm{~L}$ & -58 & 4 & -26 & 3.99 & 10 \\
\hline $\mathrm{TP}$ & $21 / 38$ & $\mathrm{R}$ & 56 & 10 & -20 & 4.11 & 8 \\
\hline VLPFC & 45 & $\mathrm{~L}$ & -52 & 32 & 0 & 5.06 & 103 \\
\hline VLPFC & 44 & $\mathrm{~L}$ & -48 & 14 & 18 & 5.15 & 94 \\
\hline HCMP & & $\mathrm{L}$ & -16 & -28 & -4 & 4.94 & 176 \\
\hline HCMP & & $\mathrm{R}$ & 18 & -30 & -2 & 4.21 & \\
\hline Lingual gyrus & $17 / 18$ & $\mathrm{~L}$ & -10 & -90 & -14 & 6.30 & \\
\hline
\end{tabular}

Study 2

\begin{tabular}{|c|c|c|c|c|c|c|c|}
\hline Phrases (Korean) & Brodmann's Area & Laterality & $x$ & $y$ & $z$ & $t$ & Vox \\
\hline DMPFC & $8 / 9$ & $\mathrm{~L}$ & -8 & 54 & 48 & 4.83 & 27 \\
\hline pSTS & 22 & $\mathrm{~L}$ & -60 & -26 & 8 & 9.64 & 381 \\
\hline pSTS & 22 & $\mathrm{R}$ & 66 & -14 & -4 & 7.96 & 276 \\
\hline $\mathrm{TP}$ & 38 & $\mathrm{~L}$ & -50 & 18 & -28 & 4.21 & 19 \\
\hline $\mathrm{TP}$ & 38 & $\mathrm{R}$ & 54 & 16 & -22 & 4.09 & 21 \\
\hline VLPFC & 45 & $\mathrm{~L}$ & -58 & 28 & 14 & 12.07 & 43 \\
\hline VLPFC & 45 & $\mathrm{~L}$ & -56 & 26 & 18 & 7.02 & 195 \\
\hline VLPFC & 47 & $\mathrm{~L}$ & -44 & 48 & -16 & 7.74 & 14 \\
\hline HCMP & & $\mathrm{L}$ & -20 & -30 & -2 & 5.47 & 98 \\
\hline HCMP & & $\mathrm{R}$ & 26 & -28 & -2 & 5.02 & 43 \\
\hline Lingual gyrus & 17 & $\mathrm{~L}$ & -18 & -88 & -16 & 10.44 & 505 \\
\hline
\end{tabular}

Study 3

\begin{tabular}{|c|c|c|c|c|c|c|c|}
\hline Video (American) & Brodmann's Area & Laterality & $x$ & $y$ & $z$ & $t$ & Vox \\
\hline DMPFC & 9 & $\mathrm{~L}$ & -14 & 54 & 40 & 3.72 & 14 \\
\hline DMPFC & $8 / 6$ & & -2 & 24 & 60 & 4.53 & 163 \\
\hline pSTS & 22 & $\mathrm{~L}$ & -54 & -40 & 2 & 4.46 & 338 \\
\hline pSTS & 22 & $\mathrm{R}$ & 50 & -36 & 0 & 4.55 & 199 \\
\hline TP & $21 / 38$ & $\mathrm{~L}$ & -54 & 6 & -28 & 4.36 & 15 \\
\hline $\mathrm{TP}$ & $21 / 38$ & $\mathrm{R}$ & 50 & 12 & -30 & 1.98 & 103 \\
\hline VLPFC & 47 & $\mathrm{~L}$ & -52 & 20 & -2 & 3.91 & 76 \\
\hline VMPFC & 11 & & -4 & 56 & -12 & 3.72 & 60 \\
\hline VMPFC & 11 & & 2 & 26 & -22 & 4.58 & 44 \\
\hline
\end{tabular}


Table 1. (continued)

\begin{tabular}{|c|c|c|c|c|c|c|c|}
\hline \multicolumn{8}{|c|}{ Study 1} \\
\hline Phrases (American) & Brodmann's Area & Laterality & $x$ & $y$ & $z$ & $t$ & Vox \\
\hline Inferior parietal lobe & 40 & $\mathrm{R}$ & 36 & -46 & 48 & 8.13 & 406 \\
\hline Inferior parietal lobe & 40 & $\mathrm{~L}$ & -40 & -58 & 48 & 4.22 & 12 \\
\hline Insula & 13 & $\mathrm{~L}$ & -34 & 12 & -4 & 6.17 & 385 \\
\hline Middle frontal gyrus & 8 & $\mathrm{R}$ & 48 & 20 & 42 & 5.28 & 109 \\
\hline Middle temporal Gyrus & 39 & $\mathrm{R}$ & 52 & -74 & 14 & 5.07 & 62 \\
\hline Middle temporal gyrus & $37 / 21$ & $\mathrm{R}$ & 58 & -62 & -2 & 4.78 & 73 \\
\hline Postcentral gyrus & $3 / 1 / 2$ & $\mathrm{R}$ & 44 & -22 & 36 & 4.58 & 7 \\
\hline Precuneus & 7 & $\mathrm{~L}$ & -16 & -46 & 50 & 4.39 & 15 \\
\hline Precuneus & $7 / 31$ & $\mathrm{R}$ & 2 & -50 & 44 & 4.78 & 117 \\
\hline Precuneus & 7 & $\mathrm{~L}$ & -8 & -68 & 42 & 6.31 & 244 \\
\hline SMA & 6 & $\mathrm{R}$ & 4 & 22 & 64 & 6 & 89 \\
\hline Superior frontal gyrus & 10 & $\mathrm{R}$ & 28 & 48 & 8 & 4.25 & 8 \\
\hline Superior frontal gyrus & 6 & $\mathrm{~L}$ & -16 & 4 & 66 & 4.06 & 8 \\
\hline Superior frontal gyrus/middle frontal gyrus & 9 & $\mathrm{R}$ & 20 & 42 & 34 & 9.02 & 278 \\
\hline Superior occipital & 19 & $\mathrm{R}$ & 44 & -82 & 26 & 5.04 & 42 \\
\hline Superior occipital & 19 & $\mathrm{~L}$ & -38 & -90 & 22 & 5.22 & 55 \\
\hline Superior parietal & 5 & $\mathrm{~L}$ & -24 & -52 & 72 & 4.44 & 15 \\
\hline Supramarginal gyrus & 40 & $\mathrm{~L}$ & -56 & -28 & 34 & 4.68 & 71 \\
\hline VLPFC & 47 & $\mathrm{R}$ & 44 & 36 & -6 & 6.72 & 70 \\
\hline
\end{tabular}

Study 2

\begin{tabular}{|c|c|c|c|c|c|c|c|}
\hline Phrases (Korean) & Brodmann's Area & Laterality & $x$ & $y$ & $z$ & $t$ & Vox \\
\hline Inferior parietal lobe & 40 & $\mathrm{~L}$ & -38 & -48 & 42 & 5.04 & 36 \\
\hline Inferior temporal gyrus & 20 & $\mathrm{R}$ & 54 & -26 & -28 & 4.3 & 15 \\
\hline Insula & 13 & $\mathrm{~L}$ & -36 & 12 & 12 & 4.27 & 10 \\
\hline Insula & 13 & $\mathrm{R}$ & 42 & 4 & 4 & 5.75 & 314 \\
\hline Middle frontal gyrus & 46 & $\mathrm{R}$ & 28 & 40 & 32 & 4.52 & 50 \\
\hline Middle frontal gyrus & $10 / 46$ & $\mathrm{~L}$ & -36 & 50 & 10 & 7.83 & 205 \\
\hline Middle frontal gyrus & 9 & $\mathrm{~L}$ & -30 & 46 & 34 & 6.89 & 250 \\
\hline Middle occipital & $19 / 39$ & $\mathrm{~L}$ & -36 & -88 & 32 & 7.32 & 143 \\
\hline Middle temporal gyrus & 39 & $\mathrm{R}$ & 42 & -70 & 16 & 10.02 & 451 \\
\hline OFC & $11 / 47$ & $\mathrm{R}$ & 24 & 30 & -18 & 4.66 & 18 \\
\hline OFC & 47 & $\mathrm{R}$ & 32 & 12 & -26 & 4.55 & 69 \\
\hline SMA & 6 & $\mathrm{R}$ & 18 & 10 & 66 & 5.33 & 114 \\
\hline Superior frontal gyrus & 10 & $\mathrm{R}$ & 20 & 66 & 10 & 4.41 & 18 \\
\hline Superior frontal sulcus & 8 & $\mathrm{R}$ & 26 & 24 & 40 & 4.46 & 61 \\
\hline
\end{tabular}


Table 1. (continued)

\begin{tabular}{|c|c|c|c|c|c|c|c|}
\hline \multicolumn{8}{|c|}{ Study 2} \\
\hline Phrases (Korean) & Brodmann's Area & Laterality & $x$ & $y$ & $z$ & $t$ & Vox \\
\hline $\mathrm{TP}$ & 38 & $\mathrm{~L}$ & -40 & 10 & -20 & 7.52 & 453 \\
\hline VLPFC & $10 / 46$ & $\mathrm{R}$ & 40 & 48 & 2 & 4.88 & 84 \\
\hline \multicolumn{8}{|c|}{ Study 3} \\
\hline Video (American) & Brodmann's Area & Laterality & $x$ & $y$ & $z$ & $t$ & Vox \\
\hline Calcarine & 30 & $\mathrm{~L}$ & -18 & -56 & 12 & 3.63 & 10 \\
\hline Fusiform/parahippocampal gyrus & 36 & $\mathrm{R}$ & 20 & -40 & -12 & 3.6 & 6 \\
\hline Inferior occipital & 19 & $\mathrm{~L}$ & -34 & -88 & 24 & 5.12 & 242 \\
\hline Middle occipital gyrus & 19 & $\mathrm{R}$ & 52 & -74 & 6 & 6.52 & 286 \\
\hline Posterior cingulate & 31 & $\mathrm{~L}$ & -16 & -24 & 44 & 4.17 & 108 \\
\hline Precuneus & $5 / 7$ & $\mathrm{R}$ & 10 & -44 & 58 & 4.86 & 220 \\
\hline Supramarginal gyrus & 40 & $\mathrm{R}$ & 58 & -26 & 34 & 4.01 & 37 \\
\hline
\end{tabular}

DMPFC $=$ dorsomedial prefrontal cortex; pSTS $=$ posterior superior temporal sulcus; TP $=$ temporal pole; VLPFC $=$ ventrolateral prefrontal cortex; HCMP $=$ hippocampus; VMPFC $=$ ventromedial prefrontal cortex; OFC = orbito-frontal cortex; Amer $=$ American participants; Kor $=$ Korean participants; vox $=$ number of voxels in cluster.

region significantly activated to persuasive, relative to unpersuasive, messages in one sample that was not significantly activated in the other sample. A conjunction analysis also confirmed that there was overlap in all key regions at $p<.005$, uncorrected (Table 2 ).

\section{Cross-cultural Differences}

Examining individual differences that commonly differ by cultural group, we found that the American sample was higher in independence $[$ mean american $=5.15$,
Figure 1. Neural regions that were more active during persuasive than unpersuasive passages in Study 1 (Americans, text-based messages), Study 2 (Koreans, text-based messages), and Study 3 (Americans, videobased messages). For display purposes, all activations in this figure use a threshold of $p=$ .005 , uncorrected. Note: Korean activations were statistically equivalent in many of the displayed regions but appear weaker because the color scales are different (see scales on left). Also, only a small portion of the actual VLPFC cluster appears in axial slice selected for the Korean sample. As shown in the Table 1A, the spatial extent of these activations is comparable. DMPFC $=$ dorsomedial prefrontal cortex; pSTS = posterior superior temporal sulcus; $\mathrm{TP}=$ temporal pole; HCMP = hippocampus; VLPFC $=$ ventrolateral prefrontal cortex.

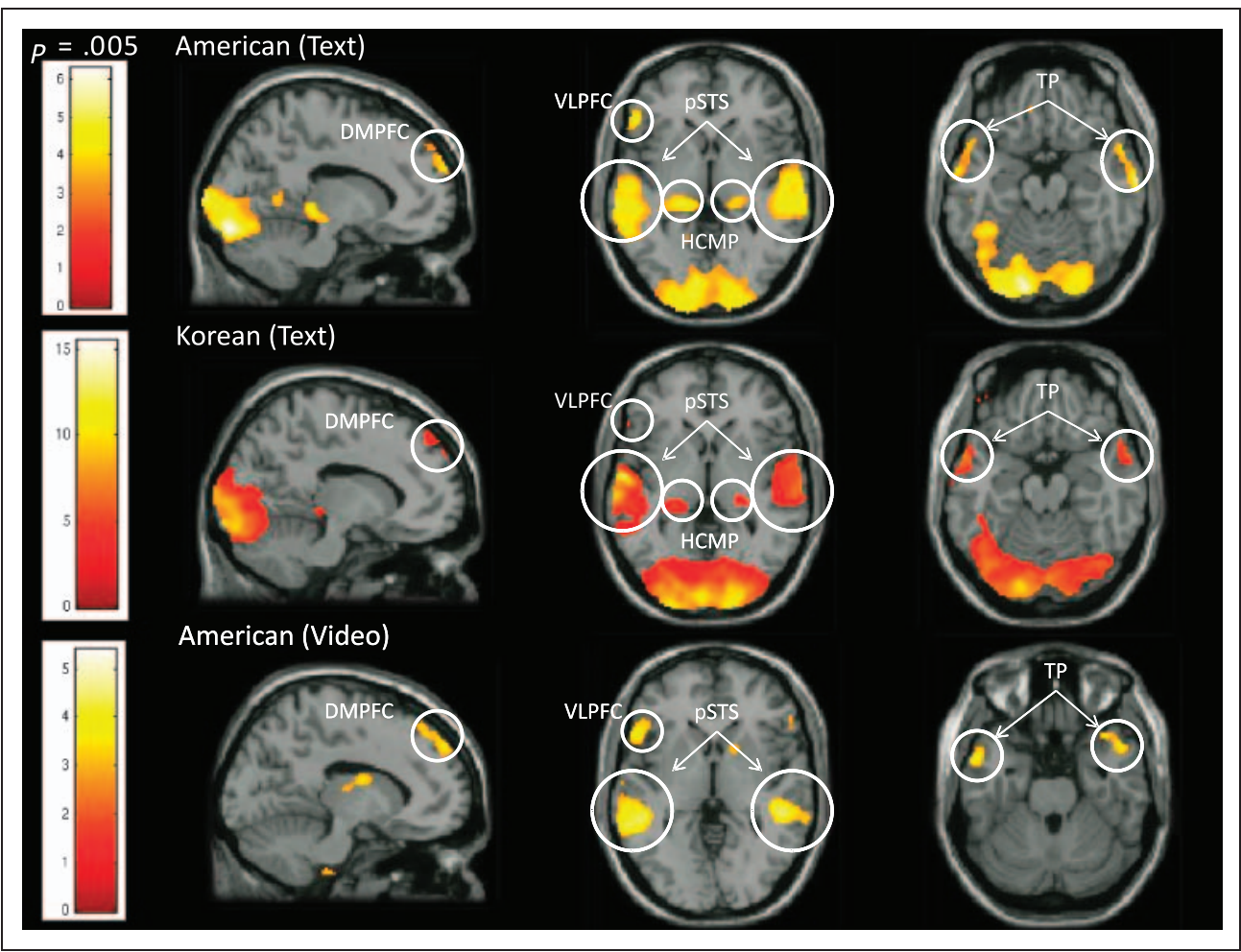


Table 2. Results of Conjunction Analysis of Activations in Studies 1 and 2, Run at $p<.005$, Uncorrected for Each Analysis

\begin{tabular}{|c|c|c|c|c|c|c|}
\hline Region & Brodmann's Area & Laterality & $x, y, z(\operatorname{Max})$ & $t$ (Amer) & $t$ (Kor) & Vox \\
\hline DMPFC & $8 / 9$ & $\mathrm{~L}$ & -126036 & 3.54 & 3.37 & 29 \\
\hline DMPFC & $9 / 10$ & $\mathrm{~L}$ & -105244 & 3.10 & 3.44 & 10 \\
\hline Lateral temporal cortex & 21 & $\mathrm{~L}$ & $-64-22-2$ & 4.23 & 9.73 & 312 \\
\hline $\mathrm{TP}$ & $21 / 38$ & $\mathrm{~L}$ & $-568-18$ & 3.33 & 7.26 & 142 \\
\hline pSTS & 22 & $\mathrm{~L}$ & $-56-308$ & 4.00 & 9.13 & 482 \\
\hline pSTS & 22 & $\mathrm{R}$ & $68-14-6$ & 4.60 & 7.75 & 214 \\
\hline Lateral temporal cortex & 21 & $\mathrm{R}$ & $58-4-6$ & 3.58 & 8.93 & 282 \\
\hline $\mathrm{TP}$ & $21 / 38$ & $\mathrm{R}$ & $604-16$ & 3.34 & 6.68 & 114 \\
\hline VLPFC & 45 & $\mathrm{~L}$ & -582810 & 4.16 & 6.12 & 264 \\
\hline HCMP & & $\mathrm{L}$ & $-16-30-2$ & 4.91 & 5.10 & 216 \\
\hline HCMP & & $\mathrm{R}$ & $24-26-4$ & 3.86 & 4.97 & 71 \\
\hline Precentral gyrus & 6 & $\mathrm{~L}$ & -52050 & 4.34 & 8.08 & 346 \\
\hline Middle occipital gyrus & 18 & $\mathrm{~L}$ & $-18-1064$ & 5.19 & 15.47 & 274 \\
\hline Cuneus & 18 & $\mathrm{R}$ & $24-100-8$ & 5.12 & 12.83 & 279 \\
\hline Lingual gyrus & 18 & $\mathrm{~L}$ & $-14-90-16$ & 6.02 & 9.93 & 443 \\
\hline
\end{tabular}

mean_korean $=4.48, t(27)=2.88, p<.01]$, and horizontal individualism $[$ mean_american $=6.73$, mean_korean $=$ $6.13, t(27)=2.28, p<.05]$, whereas the Korean group was higher in vertical collectivism [mean_american $=5.02$, mean_korean $=6.09, t(27)=2.85, p<. \overline{0} 1]$. Group means for measures of interdependence (mean_american $=4.76$, mean_korean $=5.13$ ) and vertical individualism (mean american $=5.61$, mean_korean $=5.30$ ) were in the expected direction, but were not statistically significant at $p<.05$.

Examining behavioral responses to the persuasive messages, the correlation across average block persuasiveness ratings followed a similar pattern between groups $(r=.83)$, as did the average information ratings $(r=.85)$. Furthermore, none of the average persuasion ratings for a block differed across groups at $p<.05$ (see Table 3A). A pairedsamples $t$ test (pairing across items) also suggested that there were no significant differences in average persuasion $[t(19)=1.41, p=n s]$ or information ratings $[t(19)=1.72$, $p=n s]$ across samples. Although the average block emotion scores were also highly correlated between samples $(r=.75)$, on average, Korean participants rated the arguments as more emotional than did the American participants $[t(19)=2.81, p=.01]$.

Comparing neural activation in the two samples, although the same set of brain regions were active in the American and Korean samples, there were statistical differences in activity when the samples were directly compared to one another. A variety of areas were more active in American participants (compared to Korean participants) when viewing arguments that were later rated as persuasive (compared to those that were rated as unpersuasive). These included areas that are typically implicated in emotion processing (amygdala, ventral striatum), social cognition (pSTS, posterior cingulate cortex), and memory encoding (medial temporal lobe; see Table 4; Figure 2). In examining areas that were more active in Korean participants (compared to American participants) for persuasive (compared to unpersuasive arguments), the only regions showing increased activity were in areas of inferior occipital cortex associated with visual processing.

\section{MATERIALS AND METHODS (STUDY 3)}

In addition to replicating across culturally diverse groups, we explored whether the results would replicate across stimulus modality (i.e., beyond text-based persuasive messages). Therefore, in a third study, we measured BOLD signal as participants viewed a series of video-based commercials. The design and the analysis of this study differed from the first two in the following ways: in terms of design, participants viewed professionally developed video-based commercials as persuasive stimuli instead of text-based messages, and participants rated how persuasive they found each video immediately after seeing the clip instead of waiting to exit the scanner as they had in Studies 1 and 2; in terms of analysis, we interrogated specific regions based on the activations reported above in addition to whole-brain analyses. This analysis was motivated by the strong similarity in the activations observed in Studies 1 and 2, and tested whether the same discrete network of brain regions were associated with persuasion across stimulus modality and diverse participant samples. To begin to test this, in Study 3, 
Table 3. Behavioral Ratings of Stimuli on Persuasion, Emotion and Information Dimensions

\begin{tabular}{|c|c|c|c|c|c|c|c|c|c|c|c|c|}
\hline & \multicolumn{6}{|c|}{ Americans } & \multicolumn{6}{|c|}{ Koreans } \\
\hline & $\begin{array}{l}\text { Avg } \\
\text { Pers }\end{array}$ & $\begin{array}{c}\text { Std } \\
\text { Pers }\end{array}$ & $\begin{array}{l}\text { Avg } \\
\text { Info }\end{array}$ & $\begin{array}{l}\text { Std } \\
\text { Info }\end{array}$ & $\begin{array}{l}\text { Avg } \\
\text { Emo }\end{array}$ & $\begin{array}{l}\text { Std } \\
\text { Emo }\end{array}$ & $\begin{array}{l}\text { Avg } \\
\text { Pers }\end{array}$ & $\begin{array}{c}\text { Std } \\
\text { Pers }\end{array}$ & $\begin{array}{l}\text { Avg } \\
\text { Info }\end{array}$ & $\begin{array}{l}\text { Std } \\
\text { Info }\end{array}$ & $\begin{array}{l}\text { Avg } \\
\text { Emo }\end{array}$ & $\begin{array}{c}\text { Std } \\
\text { Emo }\end{array}$ \\
\hline Topic1 & 2.33 & 0.87 & 1.53 & 0.81 & 3.87 & 0.50 & 2.50 & 0.63 & 2.00 & 0.66 & 3.21 & 0.77 \\
\hline Topic2 & 3.53 & 0.81 & 2.80 & 0.98 & 3.80 & 0.40 & 3.43 & 0.62 & 3.00 & 0.76 & 3.64 & 0.61 \\
\hline Topic3 & 3.13 & 0.62 & 2.20 & 0.75 & 3.40 & 0.71 & 2.86 & 0.74 & 2.86 & 0.64 & 2.79 & 0.86 \\
\hline Topic4 & 1.93 & 1.00 & 2.93 & 1.00 & 1.87 & 0.81 & 2.71 & 1.10 & 3.00 & 0.66 & 2.64 & 0.90 \\
\hline Topic 5 & 3.73 & 0.57 & 3.47 & 0.50 & 2.93 & 1.00 & 3.64 & 0.48 & 3.57 & 0.50 & 3.36 & 0.72 \\
\hline Topic6 & 2.87 & 0.81 & 3.33 & 0.60 & 2.00 & 0.90 & 3.00 & 0.54 & 3.43 & 0.62 & 2.50 & 1.18 \\
\hline Topic7 & 2.20 & 0.83 & 3.27 & 0.85 & 1.27 & 0.44 & 2.79 & 1.08 & 3.14 & 0.92 & 2.57 & 1.30 \\
\hline Topic8 & 3.80 & 0.40 & 3.60 & 0.61 & 2.33 & 0.79 & 3.71 & 0.45 & 3.79 & 0.41 & 3.00 & 1.07 \\
\hline Topic9 & 3.38 & 0.48 & 3.56 & 0.50 & 2.13 & 0.93 & 3.07 & 0.59 & 3.36 & 0.72 & 2.86 & 0.92 \\
\hline Topic10 & 1.88 & 0.86 & 3.00 & 0.87 & 1.56 & 0.86 & 2.57 & 0.90 & 2.71 & 0.88 & 2.00 & 1.00 \\
\hline Topic11 & 3.25 & 0.56 & 2.25 & 0.83 & 3.50 & 0.61 & 3.43 & 0.50 & 3.07 & 0.46 & 3.36 & 0.72 \\
\hline Topic12 & 3.33 & 0.60 & 3.47 & 0.62 & 2.87 & 0.81 & 3.43 & 0.50 & 3.57 & 0.62 & 2.79 & 0.86 \\
\hline Topic13 & 3.20 & 0.83 & 3.40 & 0.49 & 2.07 & 0.77 & 3.14 & 0.52 & 3.36 & 0.72 & 2.36 & 0.97 \\
\hline Topic14 & 3.50 & 0.61 & 3.44 & 0.50 & 2.38 & 1.11 & 3.43 & 0.50 & 3.57 & 0.62 & 2.86 & 0.99 \\
\hline Topic15 & 3.13 & 0.62 & 3.47 & 1.03 & 1.73 & 0.68 & 2.57 & 0.98 & 3.07 & 1.10 & 2.43 & 1.12 \\
\hline Topic16 & 2.53 & 0.96 & 3.53 & 0.50 & 1.40 & 0.61 & 2.93 & 0.88 & 3.36 & 0.61 & 2.29 & 0.96 \\
\hline Topic17 & 3.60 & 0.49 & 3.60 & 0.49 & 2.33 & 1.01 & 3.79 & 0.41 & 3.79 & 0.41 & 3.36 & 1.11 \\
\hline Topic18 & 3.07 & 0.77 & 2.80 & 0.65 & 3.07 & 0.57 & 3.21 & 0.41 & 3.29 & 0.59 & 2.57 & 0.73 \\
\hline Topic19 & 3.19 & 0.95 & 3.25 & 0.66 & 2.81 & 0.88 & 3.00 & 0.85 & 3.29 & 0.80 & 3.00 & 0.54 \\
\hline Topic20 & 2.00 & 0.82 & 2.53 & 0.81 & 1.80 & 1.11 & 2.64 & 1.04 & 2.57 & 0.90 & 2.43 & 1.05 \\
\hline \multicolumn{13}{|c|}{ B. Behavioral Responses, Video-based Messages } \\
\hline & \multicolumn{2}{|c|}{$\begin{array}{c}\text { Avg } \\
\text { Persuasive }\end{array}$} & \multicolumn{2}{|c|}{$\begin{array}{c}\text { Std } \\
\text { Persuasive }\end{array}$} & \multicolumn{2}{|c|}{$\begin{array}{c}\text { Avg } \\
\text { Emotional }\end{array}$} & \multicolumn{2}{|c|}{$\begin{array}{c}\text { Std } \\
\text { Emotional }\end{array}$} & \multicolumn{2}{|c|}{$\begin{array}{c}\text { Avg } \\
\text { Informative }\end{array}$} & \multicolumn{2}{|c|}{$\begin{array}{c}\text { Std } \\
\text { Informative }\end{array}$} \\
\hline Video 1 & \multicolumn{2}{|c|}{2.81} & \multicolumn{2}{|c|}{0.83} & \multicolumn{2}{|c|}{2.81} & \multicolumn{2}{|c|}{0.88} & \multicolumn{2}{|c|}{2.63} & \multicolumn{2}{|c|}{0.74} \\
\hline Video 2 & \multicolumn{2}{|c|}{3.11} & \multicolumn{2}{|c|}{1.01} & \multicolumn{2}{|c|}{3.22} & \multicolumn{2}{|c|}{0.75} & \multicolumn{2}{|c|}{2.89} & \multicolumn{2}{|c|}{1.01} \\
\hline Video 3 & \multicolumn{2}{|c|}{2.44} & \multicolumn{2}{|c|}{0.93} & \multicolumn{2}{|c|}{2.90} & \multicolumn{2}{|c|}{0.96} & & & & \\
\hline Video 4 & & & & & & & & & & & & \\
\hline Video 5 & & & & & & & & & & & & \\
\hline Video 6 & & & & & & & & & & & & \\
\hline Video 7 & & & & & & & & & & & & \\
\hline Video 8 & & & & & & & & & & & & \\
\hline Video 9 & & & & & & & & & & & & \\
\hline Video 10 & & & & & & & & & & & & \\
\hline Video 11 & & & & & & & & & & & & \\
\hline
\end{tabular}

Average persuasion, information and emotion ratings for messages presented. Pers = persuasive; Info = informative; Emo = emotional. 
Table 4. Regional Differences between the American Sample and the Korean Sample for Persuasive Relative to Unpersuasive Arguments

\begin{tabular}{|c|c|c|c|c|c|c|c|}
\hline (Hi > Lo Persuasive) & Brodmann's Area & Laterality & $x$ & $y$ & $z$ & $t$ & Vox \\
\hline \multicolumn{8}{|l|}{ American > Korean } \\
\hline Amygdala & & $\mathrm{L}$ & -13 & 0 & -20 & 3.77 & 47 \\
\hline Middle temporal gyrus & 22 & $\mathrm{R}$ & 56 & -64 & 14 & 4.28 & 146 \\
\hline Medial temporal lobe & $36 / 37$ & $\mathrm{R}$ & 22 & -38 & -14 & 4.25 & 43 \\
\hline Medial temporal lobe & 37 & $\mathrm{~L}$ & -34 & -42 & -14 & 3.93 & 36 \\
\hline Posterior cingulate & $5 / 31$ & $\mathrm{R}$ & 14 & -34 & 62 & 4.45 & 119 \\
\hline Precentral gyrus & 44 & $\mathrm{~L}$ & -48 & 0 & 20 & 3.94 & 11 \\
\hline Precentral gyrus & 6 & $\mathrm{R}$ & 14 & -14 & 74 & 3.74 & 25 \\
\hline Precentral gyrus & 4 & $\mathrm{~L}$ & -6 & -40 & 66 & 4.41 & 44 \\
\hline Postcentral gyrus & 40 & $\mathrm{~L}$ & -54 & -40 & 56 & 3.86 & 15 \\
\hline Postcentral gyrus & 43 & $\mathrm{~L}$ & -66 & -18 & 20 & 3.66 & 5 \\
\hline Supramarginal gyrus & 40 & $\mathrm{~L}$ & -56 & -28 & 26 & 3.67 & 6 \\
\hline pSTS & $39 / 22$ & $\mathrm{R}$ & 44 & -56 & 16 & 4.54 & 141 \\
\hline SubgenACC & 25 & $\mathrm{R}$ & 8 & 22 & -12 & 3.88 & 16 \\
\hline Superior frontal gyrus & 8 & $\mathrm{R}$ & 24 & 28 & 46 & 3.53 & 656 \\
\hline Superior occipital gyrus & 39 & $\mathrm{~L}$ & -48 & -80 & 24 & 4.03 & 85 \\
\hline Ventral striatum & & & 4 & 14 & -4 & 4.10 & 41 \\
\hline \multicolumn{8}{|l|}{ Korean $>$ American } \\
\hline Middle occipital gyrus & 18 & $\mathrm{~L}$ & -28 & -88 & -4 & 3.88 & 61 \\
\hline Inferior occipital gyrus & 18 & $\mathrm{R}$ & 40 & -90 & -12 & 4.29 & 68 \\
\hline Middle occipital gyrus & 18 & $\mathrm{R}$ & 30 & -92 & 8 & 3.83 & 52 \\
\hline IOC & 18 & $\mathrm{~L}$ & -28 & -90 & 10 & 4.08 & 67 \\
\hline
\end{tabular}

It should be noted that these are relative activations across groups, and thus, may reflect the difference between two within-group deactivations (thresholded at $p<.001$, uncorrected, 5-voxel extent).

pSTS $=$ posterior superior temporal sulcus; SubgenACC $=$ subgenual anterior cingulate cortex.

we created a set of regions of interest (ROIs) based on functional responses during Study 1 and examined the relationship of activity in those regions to persuasion in Study 3.

\section{Participants (Study 3)}

Twenty-seven European-American participants (15 women, mean age $=20.11$ years, $S D=2.66$ ) were recruited from the UCLA subject pool and through mass emails and posted fliers, and received either course credit or financial compensation for their participation. Participants met identical exclusion and safety criteria as in Study 1.

\section{Materials (Study 3)}

Widely viewed commercials were piloted to develop a final set of test videos. All videos were selected to be highly comprehensible, to range in level of persuasiveness, and pertain to objects and activities about which people were likely to have weak initial attitudes.

\section{Procedure (Study 3)}

While in an fMRI scanner, each participant viewed all commercials arranged into two runs, with order of the runs counterbalanced across subjects. Commercials ranged from 30 to $75 \mathrm{sec}$, and were separated by a $15-\mathrm{sec}$ fixation-cross period. Participants were instructed to watch each video, and were told that they would later be asked some questions about what they had seen. Directly following each video clip, participants were asked to rate whether the clip was persuasive on a 4-point scale (PERSUASIVE: $1=$ Not at all, $4=$ Definitely). Equivalent ratings were also made for informative and emotional. 


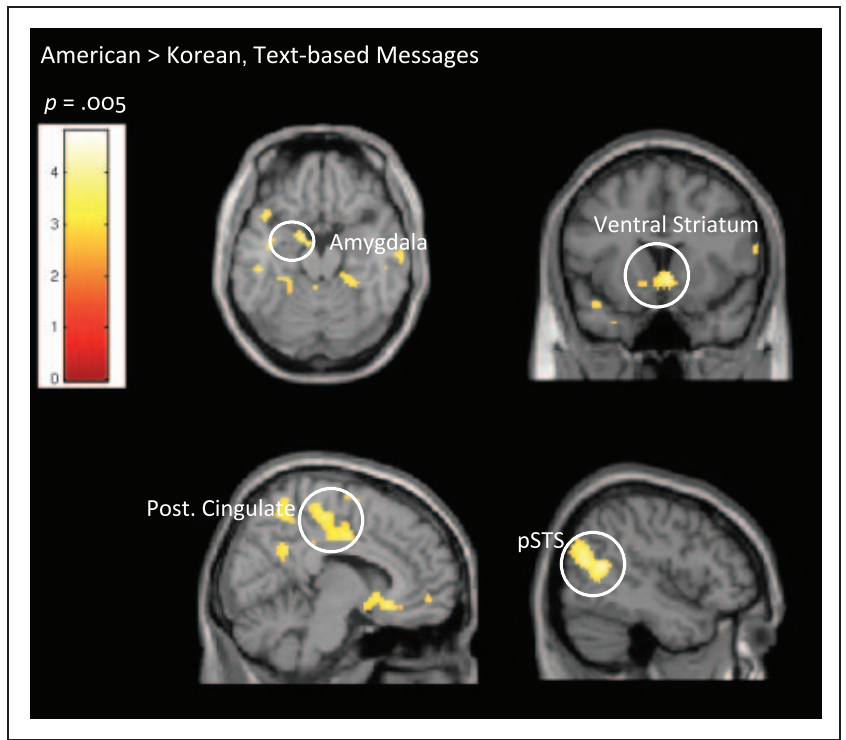

Figure 2. Neural regions that were more active in American participants than in Korean participants for persuasive compared to unpersuasive arguments. For display purposes, all activities in this figure use a threshold of $p=.005$, uncorrected. pSTS $=$ posterior superior temporal sulcus; Post. Cingulate $=$ posterior cingulate

\section{Data Acquisition and Analysis}

Imaging data were acquired using the same physical setup and imaging parameters as described in Studies 1 and 2. Two functional runs were recorded lasting 481 and $422 \mathrm{sec}$, respectively. The data were analyzed using Statistical Parametric Mapping (SPM5; Wellcome Department of Cognitive Neurology, Institute of Neurology, London, UK). Images were realigned to correct for motion, normalized into standard stereotactic space (MNI), and smoothed with an 8-mm Gaussian kernel, full width at half maximum.

The task was modeled at the first level in two ways: first, using an ANOVA model to compare activity during the task to activity during rest, and then as a regression relating neural activity to on-line persuasiveness ratings for each video. Based on the results from Studies 1 and 2, and the prior literature linking pSTS, TP, and DMPFC to social cognition, we hypothesized that activity in this network would be associated with persuasion during Study 3 . To directly test this hypothesis, we extracted ROIs based on functional activations from Study 1 (thresholded at $p=.005$, uncorrected) that were within DMPFC, TP, and pSTS as defined by the Automated Anatomical Labeling atlas (AAL; Tzourio-Mazoyer et al., 2002). Thus, we created functionally defined ROIs based on Study 1 effects that were anatomically constrained by a priori hypotheses. For each subject, we created six ROIs (right pSTS, left pSTS, right TP, left TP, and two regions in DMPFC) that each represented the average across all voxels within the circumscribed region using Marsbar (Brett, Anton, Valabregue, \& Poline, 2002).
Lastly, in order to explore whether regions outside of the putative social cognition network were also activated in response to persuasive, compared to unpersuasive, videos, we conducted a further exploratory whole-brain analysis, using a threshold of $p<.001$, uncorrected, with a 5-voxel extent threshold. All coordinates are reported in MNI space.

\section{RESULTS (STUDY 3)}

Comparing the two American groups behaviorally, the video-based messages in Study 3 were rated as less persuasive than the text-based messages in Study 1 [mean american text $=2.98$, mean american video $=2.39 ; t(29)=$ $2.66, p<.01$ ], with the vide-o-based messages being rated as less informative [mean_american_text $=3.07$, mean american_video $=2.12 ; t(29)=4.44, p<.01]$ and more emotional [mean_american_text $=2.46$, mean_american video $=2.91 ; t(\overline{26})=1.84, p=.03]$ than the text-based messages (Table 3B). Examining the neural data, however, results from our ROI analysis revealed that activity in all regions of the social cognition network were associated with persuasion, with the exception of the ROI in left pSTS (Table 5; Figure 3). Results from our whole-brain search demonstrated that as in Studies 1 and 2, finding arguments persuasive was associated with increased activity in DMPFC, bilateral pSTS, bilateral TP, and left VLPFC (Figure 1; Table 1A). Aside from these regions, the only other region that was significantly activated in response to persuasive compared to unpersuasive videos was VMPFC, a region that has typically been associated with affective processing and implicit evaluation (Koenigs \& Tranel, 2008; Knutson, Wood, Spampinato, \& Grafman, 2006; Mcclure et al., 2004).

\section{DISCUSSION}

Taken together, these results suggest that across linguistically and culturally diverse groups, as well as across different media, a distinct set of neural regions typically invoked by mentalizing tasks is associated with the experience of persuasion.

Table 5. Results of ROI Analyses in Study 3

\begin{tabular}{lcc}
\hline ROI & $t$ & $p$ \\
\hline Right pSTS & 1.65 & .056 \\
Left pSTS & 0.47 & .320 \\
Right TP & 2.66 & .007 \\
Left TP & 2.27 & .016 \\
DMPFC (anterior) & 2.51 & .009 \\
DMPFC (posterior) & 3.35 & .001 \\
\hline
\end{tabular}

ROIs were developed using functional activations in Study 1 that fell within the anatomically defined pSTS, TP, and DMPFC. $t$ Statistics were computed by averaging over all voxels in the ROI using Marsbar. 


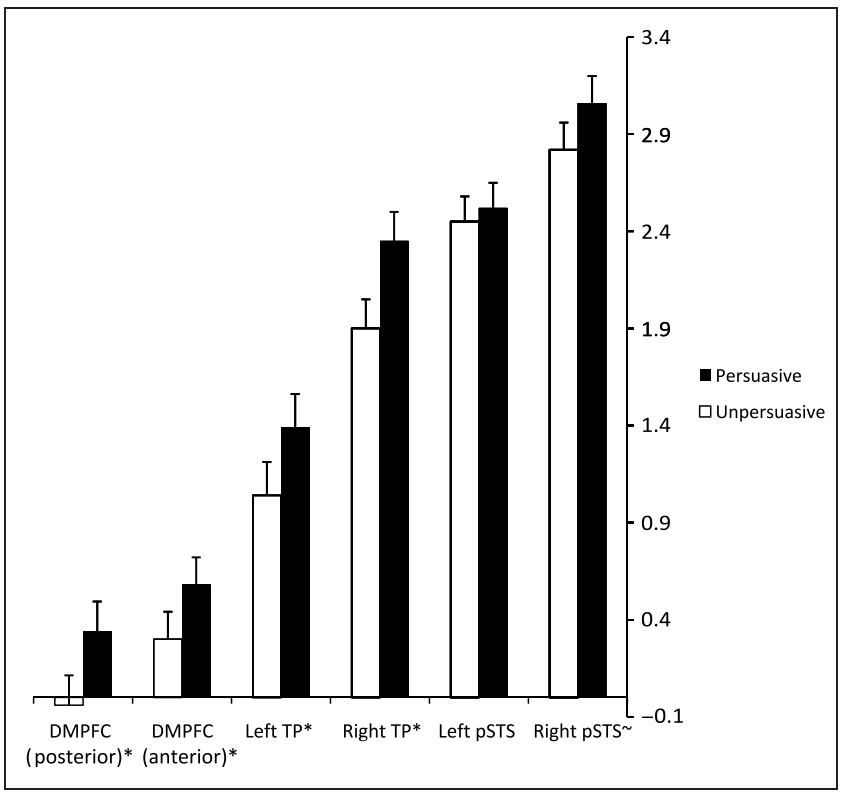

Figure 3. Mean ROI contrast values for persuasive and unpersuasive videos compared to baseline, corresponding to ROIs reported in Table 5 Note: Error bars are calculated on the difference scores across subjects as these are the errors relevant to each region-specific comparison. * Denotes significant difference at $p<.05, \sim$ denotes marginally significant difference.

Moreover, using an ROI approach, nearly all mentalizing regions that were sensitive to the experience of persuasion in a text-based message task were also sensitive to the experience of persuasion in a video-based message task.

In sum, across all three studies, increased activity in DMPFC, pSTS, TP, and left VLPFC while viewing persuasive messages was associated with feeling persuaded afterward. Consistent with work documenting the neural underpinnings of expert effects (Klucharev, Smidts, \& Fernandez, 2008), persuasion was associated with increased activity in the medial temporal lobes and visual cortex in the first two studies, in which participants viewed text-based messages and made ratings following the scanner session, but not in the third study when participants viewed video-based messages and made ratings directly following each message. Persuasion was also associated with increased activity in VMPFC in the third study.

The DMPFC, pSTS, and TP have well-documented roles in social cognitive and mentalizing tasks (Frith \& Frith, 2003). The present work extends the role of this network to include the experience of persuasion. The notion that persuasion relies on a social cognition network is consistent with Emerson's proposal that the goal of persuasion "is to bring another out of his bad sense into your good sense" (Emerson, 1880). To the extent that coordinated activity in this mentalizing network reflects consideration of another person's mental state and perspective, our results suggest that Emerson may have been pretty close to the mark. Our results are also in line with prior behavioral research that has suggested a relationship between social cognition and persuasion (Campbell \& Babrow, 2004). However, most behavioral studies of persuasion have not focused directly on perspective taking as a mechanism of persuasion, and thus, these results suggest an important new direction for persuasion research.

The overlap between the brain regions associated with persuasion effects and mentalizing in Study 3 is potentially revealing about how persuasion operates. In Studies 1 and 2, there was a single voice conveying all of the arguments; however, in Study 3, there was no obvious person serving as the message source in the video advertisements. Thus, in Study 3, there was no individual to mentalize about or whose perspective to take. One intriguing prospect is that mentalizing about a particular person's beliefs, desires, and intentions is just a special case of thinking about beliefs, desires, and intentions more generally, regardless of whether they are tied to a particular individual's mind or presented as part of a more general argument. In other words, these regions may be involved in considering a point-of-view with or without a particular source. Humans are surrounded by signs and other artifacts that suggest particular beliefs (e.g., smoking is bad) without these signs referring back to a particular person who is promoting this belief. Although we typically associate perspectives and pointsof-view with individuals, content often has a perspective long after its association with the content creator is lost.

Left VLPFC was the only other region that was more active in response to persuasive compared to unpersuasive messages in all three studies. Given that mid-VLPFC (pars triangularis) was the specific region of VLPFC activated in each study, it is plausible that this region plays a role in selecting among competing beliefs and memory representations regarding the persuasion topic. This subregion of VLPFC has been regularly observed in studies of memory selection (selecting among multiple activated memory representations) and emotional reappraisal (in which a new interpretation for an event is selected over a prior interpretation) (Badre \& Wagner, 2007; Ochsner \& Gross, 2005). As persuasion involves adopting a new interpretation over an existing one, VLPFC may play a role in this selection process. Still, it is not yet clear what role VLPFC is playing in persuasion, from the current findings alone.

Our results also speak to the modulation of neural responses by message medium. Although the majority of regions observed in any one study were replicated across all three, and five out of six regions in the main mentalizing network of interest were significantly active when using ROIs from Study 1 to predict activity in Study 3, there were some differences between the responses to persuasive text-based versus video-based arguments. For example, the medial temporal lobe was observed in response to persuasive compared to unpersuasive text based messages, whereas VMPFC was observed in response to persuasive compared to unpersuasive commercials. It is possible that this difference is related to the informational 
versus emotional content of the material. VMPFC has been associated with emotional processing and the medial temporal lobe has been associated with cognitive processing. Thus, each region may have been sensitive to types of appeals that were differentially emphasized through the two media. Manipulation checks concerning the behavioral data support this distinction; the text-based messages in Studies 1 and 2 were rated as more information-based than the commercials in Study 3, whereas the commercials were rated as more feelings-based than the text appeals.

The differential activations in the medial temporal lobe and VMPFC may also reflect the temporal distance between the persuasive messaging and self-reports of persuasion. In the first two studies, persuasion was reported after leaving the scanner, and thus, encoded associations about the persuasive messages, supported by the medial temporal lobe, may have played a role in discriminating which messages would subsequently be remembered as persuasive. In contrast, in the third study, self-reports of persuasion were obtained after each message, rendering memory processes less relevant and immediate affective responses perhaps more relevant. VMPFC has been observed in multiple studies of automatic affect (Knutson et al., 2006; Kawasaki et al., 2001) and nonreflective evaluations (Koenigs \& Tranel, 2008). Indeed, the VMPFC and medial temporal lobe tradeoff is reminiscent of similar results from studies of evaluation in the "Pepsi Challenge" (Koenigs \& Tranel, 2008; Mcclure et al., 2004). In one fMRI study (Mcclure et al., 2004), soda preferences based solely on immediate experience of taste were associated with VMPFC activity, whereas soda preferences after seeing brand names, which would presumably activate previously encoded associations, were linked to medial temporal lobe activity.

Despite these differences, the results were remarkably consistent across American (Study 1) and Korean (Study 2) subjects when the same medium was used. When analyzed separately, each group activated the same set of regions as the other. This provides initial support for the generalizability of the results in the context of this type of communication. Nevertheless, when pitted against one another, some differences did emerge cross-culturally. Specifically, Americans appeared to engage brain regions involved in socioemotional processing to a greater degree than did Koreans when reading persuasive, relative to unpersuasive, messages (Table 4; Figure 2). Interestingly, Korean participants explicitly rated the arguments as more emotional than did the American participants, whereas American participants showed comparatively more activity in regions associated with affective processing (amygdala, ventral striatum). Given that there has been relatively little research on cross-cultural differences in persuasion and the fact that cultural neuroscience (Han \& Northoff, 2008; Chiao \& Ambady, 2007) is a relatively new field, the implication of these differences is unclear. Future work that specifically targets known cultural differences should help to make sense of the activation differences observed. For example, it will be of interest to explore whether the neural response to differently framed messages (e.g., individually framed vs. collectively framed messages; gain/approach framed vs. loss/avoidance framed messages) elicit differing neural responses, in parallel with behavioral studies suggesting differences along these dimensions (Uskul et al., 2009; Khaled et al., 2008; Aaker \& Williams, 1998). This will also complement interdisciplinary applications of cultural psychology to fields such as public health and health communication (Kreuter \& Mcclure, 2004).

In summary, these studies identify for the first time the neurocognitive processes occurring at the moment that persuasion occurs. Neural activations associated with feeling persuaded were almost exclusively, and repeatedly, associated with a neural network involved in mentalizing and perspective taking. Furthermore, the specific regions identified within this network that were active in response to persuasion following text-based messages also generalized to a task in which participants were persuaded by video-based commercials. Building on the baseline provided here, future work can use neuroimaging to further advance our understanding of how people are persuaded and by what means.

\section{Acknowledgments}

Funding for this work was made possible by a National Science Foundation Graduate Research Fellowship (E.F). We thank Scott Gerwehr, Shelley Taylor, Chris Frith, Traci Mann, Brett Hemenway, Shalin Pei, Mihn-Chau Do, and Chu Kim for their feedback and assistance. For generous support, we also thank the Brain Mapping Medical Research Organization, Brain Mapping Support Foundation, Pierson-Lovelace Foundation, The Ahmanson Foundation, William M. and Linda R. Dietel Philanthropic Fund at the Northern Piedmont Community Foundation, Tamkin Foundation, Jennifer Jones-Simon Foundation, Capital Group Companies Charitable Foundation, Robson Family, and Northstar Fund. This work is dedicated to the memory of Scott Gerwehr.

Reprint requests should be sent to Matthew Lieberman, Department of Psychology, UCLA, 1285 Franz Hall, Los Angeles, CA 90095-1563, or via e-mail: lieber@ucla.edu.

\section{REFERENCES}

Aaker, J. L., \& Williams, P. (1998). Empathy versus pride: The influence of emotional appeals across cultures. Journal of Consumer Research, 25, 241-261.

Albarracin, D., Johnson, B. T., \& Zanna, M. P. (Eds.) (2005). The bandbook of attitudes. Mahwah, NJ: Erlbaum.

Aristotle. (1926). The art of rhetoric/Aristotle; with an English translation by J. H. Freese. London: Loeb Classical Library/ Harvard University Press.

Badre, D., \& Wagner, A. D. (2007). Left ventrolateral prefrontal cortex and the cognitive control of memory. Neuropsychologia, 45, 2883-2901.

Brett, M., Anton, J., Valabregue, R., \& Poline, J. (2002). Region of interest analysis using an SPM toolbox. Paper presented at the The 8th International Conference on Functional Mapping of the Human Brain, June 2-6, 2002, Sendai, Japan. (Available on CD Rom, Neuroimage Vol. 16. No. 2). 
Cabeza, R., \& Nyberg, L. (2000). Imaging cognition: II. An empirical review of 275 PET and fMRI studies. Journal of Cognitive Neuroscience, 12, 1-47.

Campbell, R. G., \& Babrow, A. S. (2004). The role of empathy in responses to persuasive risk communication: Overcoming resistance to HIV prevention messages. Health Communication, 16, 159-182.

Chaiken, S., Liberman, A., \& Eagly, A. H. (1989). Heuristic and systematic information processing within and beyond the persuasion context. In J. S. Uleman and J. A. Bargh (Eds.), Unintended thought (pp. 212-252). New York: Guilford Press.

Chiao, J., \& Ambady, N. (2007). Cultural neuroscience: Parsing universality and diversity across levels of analysis. In S. Kitayama \& D. Cohen (Eds.), Handbook of cultural psychology (pp. 237-254). New York: Guilford Press.

Crano, W., \& Prislin, R. (2008). Attitudes and attitude change. New York: Psychology Press.

Eagly, A. H., \& Chaiken, S. (1993). The psychology of attitudes. Orlando, FL: Harcourt Brace Jovanovich College Publishers.

Emerson, R. W. (1880). Letters and social aims (Vol. 4). Cambridge, MA: Riverside Press.

Frith, U., \& Frith, C. D. (2003). Development and neurophysiology of mentalizing. Philosophical Transactions of the Royal Society of London, Series B, Biological Sciences, 358, 459-473.

Han, S., \& Northoff, G. (2008). Culture-sensitive neural substrates of human cognition: A transcultural neuroimaging approach. Nature Reviews Neuroscience, 9, 646-654.

Johnson, B. T., Maio, G. R., \& Smith-McLallen, A. (2005). Communication and attitude change: Causes, processes, and effects. In D. Albarracin, B. T. Johnson, \& M. P. Zanna (Eds.), The Handbook of Attitude (pp. 617-669). New Jersey: Lawrence Erlbaum Associates.

Kawasaki, H., Kaufman, O., Damasio, H., Damasio, A. R., Granner, M., Bakken, H., et al. (2001). Single-neuron responses to emotional visual stimuli recorded in human ventral prefrontal cortex. Nature Neuroscience, 4, 15-16.

Khaled, R., Ronald, F., Noble, J., \& Biddle, R. (2008). A Qualitative Study of Culture and Persuasion in a Smoking Cessation Game. Paper presented at the Persuasive Technology: Third International Conference, Oulu, Finland, June 4-6, 2008.

Klucharev, V., Smidts, A., \& Fernandez, G. (2008). Brain mechanisms of persuasion: How "expert power" modulates memory and attitudes. Social Cognitive \& Affective Neuroscience, 3, 353-366.

Knutson, K., Wood, J., Spampinato, M., \& Grafman, J. (2006). Politics on the brain: An fMRI investigation. Social Neuroscience, 1, 25-40.

Koenigs, M., \& Tranel, D. (2008). Prefrontal cortex damage abolishes brand-cued changes in cola preference. Social Cognitive \& Affective Neuroscience, 3, 1-6.

Kreuter, M., \& Mcclure, S. (2004). The role of culture in health communication. Annual Review of Public Health, 25, 439-455.

Lieberman, M. D. (2007). Social cognitive neuroscience: A review of core processes. Annual Review of Psychology, 58, 259-289.

Markus, H. R., \& Kitayama, S. (1991). Culture and the self: Implications for cognition, emotion, and motivation. Psychological Review, 98, 224-253.

Mcclure, S., Li, J., Tomlin, D., Cypert, K., Montague, L., \& Montague, P. (2004). Neural correlates of behavioral preference for culturally familiar drinks. Neuron, 44, 379-387.

Meyers-Levy, J., \& Peracchio, L. A. (1995). Moderators of the impact of self-reference on persuasion. Journal of Consumer Research, 22, 408-423.

Nisbett, R., \& Wilson, T. (1977). Telling more than we can know: Verbal reports on mental processes. Psychological Review, 84, 231-259.

Nisbett, R. E. (2003). The geography of thought: How Asians and Westerners think differently ... and why. New York: Free Press.

Ochsner, K. N., \& Gross, J. J. (2005). The cognitive control of emotion. Trends in Cognitive Sciences, 9, 242-249.

Petty, R. E., \& Cacioppo, J. T. (1986). Communication and persuasion: Central and peripheral routes to attitude change. New York: Springer-Verlag.

Singelis, T. (1994). The measurement of independent and interdependent self-construals. Personality and Social Psychology Bulletin, 20, 580-591.

Singelis, T., Triandis, H., Bhawuk, D., \& Gelfand, M. (1995). Horizontal and vertical dimensions of individualism and collectivism: A theoretical and measurement refinement. Cross-Cultural Research, 29, 240-275.

Stayman, D. M., \& Batra, R. (1991). Encoding and retrieval of ad affect in memory. Journal of Marketing Research, 28, 232-239.

Triandis, H. (1995). Individualism and collectivism. Boulder, CO: Westview.

Tzourio-Mazoyer, N., Landeau, B., Papathanassiou, D., Crivello, F., Etard, O., Delcroix, N., et al. (2002). Automated anatomical labeling of activations in SPM using a macroscopic anatomical parcellation of the MNI MRI single-subject brain. Neuroimage, 15, 273-289.

Uskul, A., Sherman, D., \& Fitzgibbon, J. (2009). The cultural congruency effect: Culture, regulatory focus, and the effectiveness of gain- vs. loss-framed health messages. Journal of Experimental Social Psychology, 45, 535-541.

Wagner, A. D., Schacter, D. L., Rotte, M., Koutstaal, W., Maril, A., Dale, A. M., et al. (1998). Building memories: Remembering and forgetting of verbal experiences as predicted by brain activity. Science, 281, 1188-1191.

Wilson, T., \& Schooler, J. (1991). Thinking too much: Introspection can reduce the quality of preferences and decisions. Journal of Personality and Social Psychology, 60, 181-192.

Zajonc, R. B., \& Markus, H. (1982). Affective and cognitive factors in preferences. Journal of Consumer Research, 9, $123-131$. 